\title{
A QUÍMICA AMBIENTAL NO BRASIL NOS 40 ANOS DA SBQ
}

\author{
Maria C. Canela ${ }^{a *}$, Anne H. Fostier ${ }^{\mathrm{b}}$ e Marco T. Grassic \\ a'Laboratório de Ciências Químicas, Centro de Ciência e Tecnologia, Universidade Estadual do Norte Fluminense Darcy Ribeiro, \\ Av. Alberto Lamego 2000, 28013-602 Campos dos Goytacazes - RJ, Brasil. \\ 'Instituto de Química, Universidade Estadual de Campinas, 13083-970 Campinas - SP, Brasil. \\ 'Departamento de Química, Universidade Federal do Paraná, 81531-980 Curitiba - PR, Brasil.
}

Recebido em 02/04/2016; aceito em 11/05/2017

\begin{abstract}
ENVIRONMENTAL CHEMISTRY IN BRAZIL IN 40 YEARS IN THE SBQ. This paper aims to present an overview of the ongoing of the Brazilian environmental chemistry over the last forty years inserted in the context of the Brazilian Chemical Society (SBQ). Initially, a brief history presents the main milestones and premise of its "birth" in the 1980s, as well as the set of major actions taken within the SBQ to consolidate and strengthen this area of research. Then, in a separated approach between atmosphere, hydrosphere and lithosphere, we present the evolution of the main topics covered in Environmental Chemistry in Brazil, as well as the contribution of the chemists in the interdisciplinary scenario that constitutes this area. Finally, an overview of the evolution of the teaching of Environmental Chemistry in Brazil is presented.
\end{abstract}

Keywords: environmental chemistry, atmosphere, hydrosphere, lithosphere,

\section{HISTÓRICO DA QUÍMICA AMBIENTAL NO BRASIL}

No Brasil, a Química Ambiental se desenvolveu a partir de um vínculo estreito com a Química Analítica, que se consolidou com base em uma escola consolidada e tradicional, com uma visão clássica sobre seu papel e voltada, em primeira instância, para o desenvolvimento de protocolos analíticos e elucidação de problemas estabelecidos dentro do laboratório. Portanto, boa parte dos primeiros químicos ambientais tinha uma forte ligação com a Química Analítica e eram poucos aqueles com experiência em trabalhos de campo e amostragem ambiental. Nos anos 1980, a pesquisa em Química Ambiental conduzida em nosso país tinha como base o monitoramento de espécies químicas presentes na hidrosfera, atmosfera e biota. ${ }^{1}$ Existem documentos evidenciando a escassez de atividades de pesquisa dedicadas ao estudo da reatividade química e comportamento de poluentes não apenas nos três compartimentos mencionados anteriormente, mas igualmente em suas interfaces. ${ }^{2}$ Estas circunstâncias resultaram em um desenvolvimento mais lento da Química Ambiental brasileira, quando comparada com a de outros países, especialmente os desenvolvidos. Não obstante estes aspectos, nos dias atuais a Química Ambiental ocupa posição de destaque no cenário científico nacional, tendo igualmente alcançado projeção internacional. Vários eventos científicos também têm contribuído para isso, tais como as Reuniões Anuais da Sociedade Brasileira de Química, SBQ, os Encontros Nacionais de Química Analítica (ENQA), o Congresso Brasileiro de Química Ambiental, posteriormente denominado International Environmental Chemistry Congress in Brazil, além dos Encontros Nacionais de Química Ambiental (ENQAmb), entre outros. Estes eventos têm representado um importante fórum de discussão, inclusive relacionada aos desafios e perspectivas da Química Ambiental no Brasil. Outros fatores relevantes foram a criação da Divisão de Química Ambiental da SBQ em 1994 e a formação de um expressivo número de pesquisadores oriundos dos grupos que se consolidaram nos anos 80 e 90 . Estes pesquisadores lideram hoje grupos atuantes distribuídos em todo o território nacional. Também deve ser citada a criação da disciplina Química Ambiental, que passou a integrar a

*e-mail: mccanela@gmail.com,mccanela@uenf.br grade curricular dos cursos de graduação em Química no Brasil a partir dos anos 1990 e 2000. ${ }^{3}$ Programas governamentais como os Institutos do Milênio e, mais recentemente, o dos Institutos Nacionais de Ciência e Tecnologia (INCT) também têm contribuído para este desenvolvimento, especialmente em função da possibilidade concreta de financiamento para o estabelecimento de redes de pesquisa. Nesse contexto pelo menos três institutos podem ser destacados, sendo eles o Instituto Nacional de Ciência e Tecnologia de Energia e Ambiente (INCT-E\&A), o Instituto Nacional de Ciências e Tecnologias Analíticas Avançadas (INCTAA) e o Instituto Nacional de Ciência e Tecnologia de Transferência de Materiais Continente-Oceano (INCT-TMOcean), com forte atuação de pesquisadores atuantes na área de química.

\section{CENÁRIO DE ESTUDO DA QUÍMICA AMBIENTAL NO BRASIL}

Para contemplar o cenário das pesquisas em Química Ambiental no Brasil, decidimos dividir os estudos em hidrosfera, atmosfera e litosfera, embora estes compartimentos sejam integrados e não haja um mais importante que o outro e tampouco podem ser vistos separadamente. Além disso, este texto não tem a pretensão de fazer uma revisão exaustiva das publicações nestas áreas dentro da Química Ambiental, mas sim de apresentar um panorama da evolução e dos temas que foram e estão sendo estudados nestes 40 anos da Sociedade Brasileira de Química, apresentando exemplos de alguns destes trabalhos.

\section{Atmosfera}

O estudo da química atmosférica no Brasil é muito mais antigo do que podemos imaginar. Segundo relatam Marques e Filgueiras, ${ }^{4}$ ela começou a ser estudada no Brasil com uma perspectiva ambiental na metade do século XVIII e início do século XIX, por um médico chamado José Pinto de Azeredo. Azeredo estudou medicina na Universidade de Edimburgo em 1786, onde redigiu uma memória sobre os efeitos do $\mathrm{CO}_{2}$, chamado antigamente de ar fixo, sobre o sistema nervoso. Marques e Filgueiras ${ }^{4}$ destacam que este trabalho foi 
pioneiro tanto no hemisfério ocidental, como no mundo, ao colocar a química à serviço da determinação da qualidade do ar e mostrar a preocupação ambiental com o ar que respiramos.

Dentro da mesma perspectiva de análises químicas para avaliação da qualidade do ar, a CETESB (Companhia Ambiental do Estado de São Paulo) iniciou o monitoramento da qualidade do ar em 1972, medindo diariamente os níveis de dióxido de enxofre $\left(\mathrm{SO}_{2}\right)$ e fumaça preta, na região Metropolitana de São Paulo. No início da década de 1980, a companhia ampliou o número de parâmetros a serem medidos, automatizou as medidas e estudos específicos começaram a ser realizados com a Universidade de São Paulo (USP), iniciando estudos de fontes, mecanismos de formação e reações na atmosfera. ${ }^{5}$

Os trabalhos relacionados à química atmosférica, não só da cidade de São Paulo, começaram a aparecer a partir da década de 1980 e sempre foram trabalhos interdisciplinares, onde estavam envolvidos tanto químicos, como físicos, biólogos, meteorologistas, agrônomos, engenheiros e médicos. Em 1981, Trindade e colaboradores ${ }^{6}$ publicaram um estudo realizado na cidade do Rio de Janeiro sobre a presença de onze metais em material particulado em suspensão coletado em diferentes locais da cidade de 1975 a 1978. O Grupo para Estudos da Poluição do Ar da USP realizou estudos de 1980 a 1982, tanto em áreas naturais na Amazônia e Goiás, como também estudaram partículas finas e grossas de aerossóis naturais (na reserva da Juréia) e urbanos de várias capitais brasileiras (Vitória, Salvador, Porto Alegre, São Paulo e Belo Horizonte). ${ }^{7,8}$ Muitos estudos estavam centrados na presença de metais tóxicos na atmosfera e que poderiam causar danos à saúde humana. Alguns trabalhos como os de Carvalho e colaboradores ${ }^{9,10}$ foram realizados por químicos em parceria com pesquisadores de departamentos de medicina. Estudos de metais na atmosfera também foram realizados na Amazônia, principalmente devido a utilização de mercúrio na extração de ouro. ${ }^{11,12}$ Também foi na década de 1980 que foram publicados os primeiros trabalhos de aldeídos na atmosfera nas cidades brasileiras, principalmente devido ao aumento na utilização de álcool como combustível após o Proálcool em 1975..$^{13,14} \mathrm{O}$ trabalho de Tanner e colaboradores, ${ }^{15}$ além de aldeídos, também estudou a presença de nitrato de peroxiacetila (PAN) e nitratos orgânicos na atmosfera urbana da cidade do Rio de Janeiro. Neste mesmo período aparecem os primeiros trabalhos com hidrocarbonetos policíclicos aromáticos no Brasil.

Finalmente em termos históricos, pesquisadores brasileiros juntamente com a NASA (U.S. National Aeronautics and Space Administration) realizaram o primeiro estudo de grandes dimensões no Brasil chamado ABLE-2 (Amazon Boundary Layer Experiment) que tinha como objetivo analisar aerossóis e gases naturais biogênicos na atmosfera Amazônica durante as estações de seca ${ }^{16,17}$ e posteriormente foi criado o ABLE-2B (1987) para estações úmidas. ${ }^{18}$

Após 40 anos de Sociedade Brasileira de Química, o que pode ser visto é que os trabalhos em Química Atmosférica envolvem diferentes grupos de pesquisa, na maior parte das vezes interdisciplinares, e que mesmo antes da criação da Divisão de Química Ambiental vários pesquisadores destes grupos já atuavam dentro da Divisão de Química Analítica, contribuindo em um primeiro momento para que as ferramentas analíticas pudessem ser usadas a favor dos estudos ambientais da química atmosférica no Brasil. ${ }^{19-25}$

Os estudos de poluentes atmosféricos em ambientes externos realizados no Brasil estão centrados em gases inorgânicos, aerossóis, material particulado e reações na atmosfera e são realizados nas grandes cidades, evidentemente devido ao maior índice de contaminação por veículos automotores e maior concentração de indústrias. Regiões metropolitanas como Rio de Janeiro, São Paulo e Salvador têm sido as mais estudadas, embora outras cidades como Cubatão, Londrina, São José dos Campos, Araraquara, Belo Horizonte, Florianópolis, Manaus, etc. também tem sido objeto de estudo devido à baixa qualidade do ar e/ou localização de grupos de pesquisa que trabalham próximos a estas cidades. A atmosfera de algumas cidades e sua vizinhança tem sido também estudada devido a poluição gerada pela queima de cana-de-açúcar ou mesmo pela atividade de mineração.

Nestes casos, os poluentes mais estudados são $\mathrm{SO}_{2}, \mathrm{NO}_{\mathrm{X}}, \mathrm{O}_{3}$, $\mathrm{NH}_{3}$, hidrocarbonetos e material particulado (PTS, $\mathrm{MP}_{10}$ e $\mathrm{MP}_{2,5}$ ), além da análise química das precipitações. A maior parte destes trabalhos visa identificar não apenas a quantidade do poluente, mas suas fontes, especiação, efeitos que os mesmos causam, localização geográfica e condições climáticas, mostrando que só a presença ou não de determinado poluente não é o único fator que interfere na má qualidade do ar. ${ }^{26-31}$ Muitos desses trabalhos fazem uma análise ambiental de dados já coletados por estações meteorológicas de órgãos ambientais estaduais ou mesmo municipais. ${ }^{26-29}$ Estudos químicos de precipitações em ambientes impactados também têm sido importantes para avaliar a contaminação atmosférica, reações químicas e fontes de contaminação. ${ }^{32-36} \mathrm{Em}$ se tratando de região metropolitana, há muitos estudos que buscam relacionar a composição química do poluente aos efeitos tóxicos à saúde humana, embora poucos tem a participação de químicos. ${ }^{37-41}$

A utilização de álcool como combustível, o aumento de emissões veiculares e também queima de biomassa fizeram com que diversos grupos de pesquisa de química ambiental começassem a trabalhar não só com compostos inorgânicos, mas também com compostos orgânicos voláteis (COV) e hidrocarbonetos policíclicos aromáticos associados ou não ao material particulado..$^{36,42-45} \mathrm{~A}$ presença dos $\mathrm{COV}$ na atmosfera, incentivou estudos fotoquímicos, especiação e reações formadoras de compostos secundários. Os principais COV encontrados em atmosferas externas são o formaldeído e o acetaldeído ${ }^{43,46,47}$, seguido por hidrocarbonetos aromáticos, como BTEX (benzeno, tolueno, etil-benzeno e xilenos) e isoprenos. ${ }^{48-50}$

Finalmente, a associação de compostos orgânicos ao material particulado é um tema bastante explorado, já que estes materiais funcionam como transportadores desses compostos, carregando-os para áreas remotas. Vários grupos têm caracterizado estes materiais, tanto em grandes cidades, ${ }^{51-53}$ como na região agrícola, uma vez que a liberação e composição do material particulado torna possível traçar fontes da queima de biomassa. ${ }^{54,55}$

No que diz respeito à Amazônia, a partir de 1995, foi criado no Brasil, o Programa LBA (Experimento de Larga Escala da BiosferaAtmosfera na Amazônia), gerenciado pelo MCTI e coordenado pelo Instituto Nacional de Pesquisas da Amazônia/INPA, com a participação de 280 instituições nacionais e estrangeiras, com a participação de vários pesquisadores brasileiros e estrangeiros (físicos, químicos, biólogos, meteorológicos, etc.). Na questão atmosférica, os pesquisadores têm estudado desde os principais gases do efeito estufa, a interferência na atmosfera das regiões alagadas, compostos orgânicos voláteis $(\mathrm{COV})$ da floresta e interação com a poluição das cidades e a ciclagem de nitrogênio. ${ }^{56-58}$ Ainda sobre a Amazônia, dentro do LBA, em 2014 foi lançado o programa de pesquisa GoAmazon (Green Ocean Amazon Experiment) que integra também a comunidade de química ambiental e tem como objetivo desenvolver as pesquisas relacionadas com a dinâmica da floresta e sua interação com a atmosfera através de experimentos e modelos climáticos. Observa-se, portanto, que os trabalhos em química ambiental desenvolvidos na Amazônia, devido à complexidade do espaço e interface, são sempre realizados por uma rede de pesquisadores, na maior parte das vezes bastante interdisciplinar. ${ }^{58-63}$

Finalmente, a química atmosférica de ambientes confinados vem também sendo explorada por químicos brasileiros, embora em menor intensidade que os ambientes externos. Segundo Brickus e Aquino Neto, ${ }^{64} \mathrm{o}$ estudo da qualidade do ar em ambientes confinados começou no Brasil na década de 1990, com químicos, microbiologistas, 
engenheiros, arquitetos e toxicologistas. Os compostos mais analisados são o $\mathrm{CO}_{2}, \mathrm{CO}$, compostos orgânicos voláteis e semi-voláteis totais e alguns especiados, material particulado, radônio e microrganismos. Grosjean, Miguel e Tavares ${ }^{65}$ realizaram um estudo pioneiro no Brasil em que mediram a presença de compostos carbonilados dentro de ambientes internos e observaram que as concentrações destes compostos nestes ambientes eram tão altas quanto as externas, sendo influenciada pelo tráfego de veículos e utilização de etanol como combustível. Mais tarde, outros trabalhos avaliaram a presença de poluentes orgânicos e inorgânicos na atmosfera de edifícios, escritórios, restaurantes e hotéis na região Sudeste do Brasil ${ }^{66-68}$ e observaram a presença de vários destes poluentes acima do limite estabelecido internacionalmente. Vários estudos envolvendo a poluição atmosférica em ambientes confinados no Brasil, com apresentação em várias Reuniões Anuais da SBQ, também foram reportados por Gioda e Aquino Neto ${ }^{69}$ em uma revisão publicada em 2003. Estudos mais sistemáticos foram realizados posteriormente, incluindo a análise de formaldeído e acetaldeído, ${ }^{70,71}$ além de compostos orgânicos voláteis totais e especiação (principalmente de benzeno, tolueno, etil-benzeno e xileno (BTEX)) e material particulado em diferentes espaços. ${ }^{72-74}$ Os locais mais estudados têm sido escolas, ${ }^{75,76}$ hospitais, ${ }^{71}$ gráficas, ${ }^{77}$ residências para idosos, ${ }^{74}$ bancos e escritórios, ${ }^{78}$ estacionamentos e túneis. ${ }^{79,80}$

\section{Hidrosfera}

Um dos primeiros trabalhos brasileiros associados à presença de espécies químicas em ambientes aquáticos data de 1972, onde resíduos de pesticidas clorados, especialmente de BHC e DDT foram encontrados em amostras de águas destinadas ao consumo humano no estado de São Paulo. ${ }^{81}$ Mais adiante, em 1979, os níveis de mercúrio total foram determinados em amostras de água e peixes coletadas no Estuário de São Vicente, igualmente em São Paulo. ${ }^{82}$ Nas duas décadas seguintes publicações envolvendo o mercúrio cresceram em número no país, especialmente em função do seu uso indiscriminado no garimpo do ouro, nas regiões centro-oeste e norte do Brasil. Pfeiffer e colaboradores ${ }^{83}$ avaliaram a presença de mercúrio em águas, sedimentos e peixes em regiões de garimpo, em Rondônia, e encontraram concentrações elevadas deste elemento em algumas espécies de peixes, com valores superiores em até cinco vezes aos recomendados para consumo humano. Outras publicações abordam a distribuição do $\mathrm{Hg}$ entre a coluna de água, o sedimento e a biota em uma área de garimpo em Poconé ${ }^{84}$ Poconé e Alta Floresta ${ }^{85}$ (MT) e nos reservatórios de usinas hidrelétricas no Pará. ${ }^{86}$ Em 1997, Lacerda publica artigo de revisão avaliando a evolução da contaminação por mercúrio no Brasil, com ênfase para as fontes industriais e garimpo do ouro. ${ }^{87}$ Outros autores realizaram estudos no estado do Amapá, no extremo norte do Brasil, determinando os níveis de mercúrio na população e em amostras ambientais e estudando os fluxos deste elemento em uma bacia localizada em área de floresta natural e em área utilizada na mineração de $\mathrm{Mn} .{ }^{88,89}$ Fadini e colaboradores ${ }^{90}$ avaliaram as fontes e o ciclo do mercúrio na bacia do Rio Negro e, a despeito das elevadas concentrações encontradas em rios, lagos, no solo e na precipitação total, concluíram que a principal contribuição para com o enriquecimento do $\mathrm{Hg}$ na região é a sua lixiviação a partir do solo. ${ }^{90}$ Lacerda e colaboradores haviam chegado a conclusões similares a partir de estudos realizados na região do Rio Madeira. ${ }^{91}$ Aspectos relacionados a fontes e ciclo do mercúrio, assim como aos processos envolvendo sua metilação, também foram e têm sido avaliados em outras regiões do país, com destaque para o Rio de Janeiro, além da própria região amazônica..$^{91,92}$

Ao longo dos últimos 40 anos, outras espécies metálicas tais como cádmio, cobre, chumbo, cromo e zinco, entre outros, também têm sido estudadas, em corpos de água doce e estuários, com especial destaque para suas respectivas fontes, ${ }^{93,94}$ distribuição, biodisponibilidade e destino. ${ }^{95-102}$ Outro elemento de interesse do ponto de vista ambiental e de saúde pública é o arsênio, que tem sido estudado em águas doces, estuarinas, sedimentos, águas de abastecimento público e mesmo em humanos. ${ }^{103-112}$

Não obstante o artigo de Lara e Barreto, ${ }^{81}$ mencionado anteriormente como pioneiro no contexto ambiental, estudos envolvendo contaminantes orgânicos têm sido conduzidos em anos mais recentes, quando comparados àqueles envolvendo espécies metálicas, em ambientes aquáticos brasileiros. A presença de hidrocarbonetos policíclicos aromáticos (HPA) em amostras de chorume de um lixão em Niterói (RJ) foi registrada em 2002. ${ }^{113}$ Hidrocarbonetos aromáticos (HPA) e alifáticos (n-alcanos) têm sido determinados frequentemente em ambientes aquáticos doces e estuarinos, em todo o território nacional, com a aplicação de estratégias voltadas para a determinação das fontes de contaminação. ${ }^{114-130}$ Em outros casos, as determinações de HPA têm sido realizadas visando a avaliação de alterações em ecossistemas aquáticos, em particular nos sedimentos. ${ }^{129,131,132}$ Outras classes de contaminantes orgânicos, como os poluentes orgânicos persistentes (POP) também têm sido estudados em ambientes aquáticos. Entre os POP podem ser mencionados os compostos organoclorados, bifenilas policloradas (PCB), DDT e DDE, entre outros. ${ }^{133-140} \mathrm{Em}$ artigo de revisão publicado em 2007 em Química Nova, Almeida e colaboradores apresentaram uma avaliação relacionada à presença de inúmeros contaminantes orgânicos, denominados substâncias tóxicas persistentes, tanto em matrizes ambientais (solos, sedimentos, água, ar e biota), quanto em matrizes tais como leite, sangue e cabelo humanos. ${ }^{141}$ Presentes até recentemente em tintas anti-incrustantes (de segunda geração) utilizadas em embarcações, os compostos organoestânicos têm sido bastante avaliados em amostras de águas e sedimentos, principalmente em ambientes aquáticos estuarinos, por estarem inequivocamente relacionados ao imposex, fenômeno que consiste no surgimento de caracteres sexuais masculinos em fêmeas de neogastrópodes. ${ }^{142-152}$

Nos anos mais recentes tem havido um crescimento dos estudos envolvendo os contaminantes químicos emergentes, ou simplesmente contaminantes emergentes, em ambientes aquáticos, dados que estas substâncias, frequentemente presentes em águas naturais e de abastecimento público, assim como de sedimentos, entre outros, não são determinadas nos programas oficiais de monitoramento da qualidade da água. Muitas destas substâncias são suspeitas de provocar interferência no sistema endócrino de organismos intactos, incluindo os seres humanos. ${ }^{153-158}$

\section{Litosfera}

A química do solo, nascida na segunda metade do século XIX, interessou-se tradicionalmente às reações químicas que ocorrem em solos e afetam a nutrição e o crescimento das plantas. ${ }^{159}$

Somente a partir da década de 1980, que a comunidade científica tomou ciência da dimensão dos problemas associados à poluição dos solos resultante de diversas atividades antrópicas (agrícolas, industriais e de mineração, deposição de resíduos sólidos, etc). Entre os principais poluentes de natureza orgânica ou inorgânica encontram-se nutrientes tais como nitrogênio e fósforo; metais e semi-metais tais como $\mathrm{Cd}, \mathrm{Cr}, \mathrm{Hg}, \mathrm{Pb}$ e As; óleos e derivados; ácidos inorgânicos e elementos radioativos ${ }^{159}$. Além disso, um número cada vez maior de contaminantes emergentes incluindo pesticidas, fármacos, ou ainda nanopartículas manufaturadas vem aumentando esta lista ao longo dos anos.

Assim como em outros compartimentos, o entendimento das reações químicas que sofrem estes contaminantes no solo constitui um 
ponto chave na previsão do seu destino e dos seus efeitos no ambiente. Entretanto, no caso do solo, devido à sua natureza trifásica (sólida/ líquida/gasosa), a dinâmica e os mecanismos de contaminação, são também intimamente ligados às condições hidrológicas e pedo/geológicas. ${ }^{159,160} \mathrm{E}$ isso explica provavelmente a alta multidisciplinaridade dos estudos inicialmente desenvolvidos em relação à contaminação dos solos, embora assim como para qualquer outro compartimento, tratando-se de Química Ambiental, uma abordagem interdisciplinar seja desejável. ${ }^{161}$

No Brasil, casos de poluição de solos e águas subterrâneas começaram a ser estudados a partir do meio da década de $1980^{162}$, mas é principalmente na década de 1990 que trabalhos começaram a ser publicados. Vários foram associados aos graves acidentes que resultaram em contaminação ambiental e humana tais como os casos de poluição por Cs-137 em Goiânia (GO) ${ }^{163,164}$ por hexaclorociclohexano em Duque de Caxias (RJ), ${ }^{165}$ ou ainda por compostos orgânicos voláteis em Porto Feliz. ${ }^{166}$ Estudos foram também desenvolvidos sobre metais provenientes da deposição de lodo de estação de tratamento de efluentes ${ }^{167,168}$ ou de resíduos sólidos industriais. ${ }^{169}$ Os riscos de poluição ambiental resultante da lixiviação dos rejeitos de mineração e da indústria de fundição começaram também a serem estudados neste período. Amaral e colaboradores ${ }^{170}$, por exemplo, estudaram os impactos ambientais dos rejeitos da mineração de Urânio iniciada em Poços de Caldas em 1982.

Como já ressaltado anteriormente, a corrida ao ouro na Amazônia, iniciada em 1975, começou a suscitar fortes preocupações ambientais na década de 80 , principalmente em relação à contaminação do ambiente por mercúrio. Entretanto, é somente na década de 90 que estudos ambientais começaram a reportar concentrações de $\mathrm{Hg}$ em solos da Amazônia ${ }^{171-173}$ e de outras regiões. ${ }^{174}$ Porém, a análise destes trabalhos revela que estes estudos foram principalmente desenvolvidos dentro da geociência, da agronomia ou mesmo da área da saúde, com baixa participação de químicos nos mesmos.

Por outro lado, no final dos anos 90, observa-se uma participação crescente dos Químicos em estudos sobre solos voltados para o estudo de processos que influem sobre a mobilidade dos poluentes (orgânicos e inorgânicos) neste compartimento ${ }^{175-177}$, sua biodisponibilidade, ${ }^{178}$ sua especiação química ${ }^{179}$, entre outros. Primeiros estudos sobre o ciclo biogeoquímica do $\mathrm{Hg}$ na região amazônica, foram também publicados nesta época, ${ }^{180}$ embora outros grupos já estavam pesquisando sobre a mesma problemática. ${ }^{90,181}$ Estudos sobre a caracterização da matéria orgânica, em particular das substâncias húmicas, e sua importância no transporte de contaminantes em solos brasileiros começam também a serem desenvolvidos por ou em colaboração com químicos. ${ }^{182,183}$

Qualquer que seja o compartimento considerado, estudos sobre poluentes (presença, transporte, reações químicas, etc) necessitam na maioria dos casos que os mesmos possam ser quantificados na matriz considerada. Dada a complexidade do solo e a baixa concentração de vários poluentes $\left(\mu \mathrm{g} \mathrm{kg}^{-1}, \mathrm{ng} \mathrm{kg}^{-1}\right)$, a quantificação de substâncias neste meio leva a uma forte demanda de desenvolvimento de métodos analíticos, tanto na parte instrumental, quanto de preparo das amostras. ${ }^{184,185}$ Neste quesito a química ambiental como um todo, mais particularmente em relação ao estudo dos solos, deve um forte tributo à química analítica. De fato, ao longo dos últimos anos observa-se um número crescente de publicações nas quais o desenvolvimento de métodos analíticos encontra-se perfeitamente integrado dentro do contexto ambiental. Embora longe de ser uma lista exaustiva, alguns trabalhos permitem exemplificar esta tendência internacional no contexto de estudos desenvolvidos sobre solos brasileiros ${ }^{166,179,182,184,186-191}$

Paralelamente à academia, agências ambientais de diferentes estados (Rio de Janeiro (FEEMA, atual INEA), São Paulo (CETESB) e Rio Grande do Sul (FEPAM) se mobilizaram para estabelecer métodos de avaliação de risco de sítios contaminados e de remediação dos mesmos. ${ }^{192}$ Este trabalho pioneiro por parte das agências teve continuação nos anos seguintes, ${ }^{193}$ e em conjunto com vários outros desenvolvidos por diferentes grupos de pesquisa, serviram de base para a elaboração, quase dez anos depois, da Resolução CONAMA 420/2009, ${ }^{194}$ a qual estabelece "critérios e valores orientadores de qualidade do solo quanto à presença de substâncias químicas e estabelece diretrizes para o gerenciamento ambiental de áreas contaminadas por essas substâncias em decorrência de atividades antrópicas". Esta resolução inclui atualmente uma lista de 20 contaminantes inorgânicos e 55 orgânicos, para os quais valores limites em solos e água subterrânea foram fixados, baseados em avaliações de riscos à saúde humana e ao meio ambiente.

Sabe-se, entretanto, que esta lista está longe de contemplar a totalidade das substâncias potencialmente tóxicas e susceptíveis de serem depositadas em solos. Além disso, inúmeras novas substâncias, de natureza xenobiótica ou não, vem sendo comercializadas anualmente sem que suas interações com o meio ambiente, e particularmente os solos, nem sua toxicidade sobre os organismos (macro e micro) tenham sido avaliadas. ${ }^{195}$ Estes, frequentemente denominados micro e nanopoluentes (em razão das suas concentrações ng ou $\mu \mathrm{g} \mathrm{kg}^{-1} \mathrm{ou}$ $\mathrm{L}^{-1}$ ) incluem, pesticidas, nanopartículas manufaturadas e fármacos, entre outros. ${ }^{196}$

Muitos estudos vêm sendo desenvolvidos sobre estes contaminantes em solos de regiões temperadas sobre sua mobilidade e sua biodisponibilidade, destinados a modelar seu comportamento e prever a sua concentração e toxicidade em solos. Entretanto, em razão de diferenças fundamentais relativas à química dos solos de regiões tropicais e subtropicais (baixa atividade das argilas minerais, baixo teor de matéria orgânica, baixo $\mathrm{pH}$, alto teor em óxido/hidróxidos de $\mathrm{Fe}$ ) estes modelos são raramente aplicáveis para solos destas regiões. ${ }^{197}$ Frente a este cenário, é, portanto, urgente que estudos sobre o comportamento de tais contaminantes sejam desenvolvidos em solos de regiões tropicais e subtropicais, tais como encontrados no Brasil. Tais estudos devem contemplar: 1) os mecanismos de interação entre o contaminante e o solo, os quais são na maioria das vezes de natureza química, e dependem tanto das propriedades físico-químicas do contaminante, quanto das propriedades do solo; 2) sua degradabilidade, seja por processos biológicos, químicos, ou fotoquímicos. Por outro lado, como já ressaltado anteriormente, estudos desta natureza necessitam que métodos analíticos, os quais incluem métodos de extração e pré-concentração, sejam previamente desenvolvidos e devidamente validados.

\section{A EVOLUÇÃO NO ENSINO DE QUÍMICA AMBIENTAL NO BRASIL NESTES 40 ANOS}

O ensino de Química Ambiental no Brasil se iniciou nos fins da década de 1980 e o curso de Química do Instituto de Química da Unicamp foi o pioneiro ao oferecer uma disciplina chamada Química Ambiental, em 1987. ${ }^{198}$ A criação desta disciplina e uma ementa adequada para ela, já antecipava a Constituição de 1988, na qual institui que o Poder Público deve inserir na questão ambiental, a Educação Ambiental em todos os níveis de ensino. Embora fazer ou ensinar Educação Ambiental não fosse o mesmo que ensinar Química Ambiental, neste momento há um impulso para que a comunidade química se volte aos processos químicos que ocorrem no ambiente, se ressalte a importância da química e os desequilíbrios que ele pode sofrer se não houver um mínimo de cuidado e conhecimento deste ambiente. Conhecendo o ambiente quimicamente, seria muito natural ensinar Educação Ambiental e preparar profissionais capacitados para preservar o ambiente. Desde então a Química Ambiental já era vista como uma disciplina Inter, Multidisciplinar e que deveria 
ser considerada de forma holística e integrada. ${ }^{3}$ No entanto, neste período não havia material em português para o ensino de Química Ambiental e muito menos artigos ou material digital para auxiliar o aluno a aprender ou o professor a ensinar. Após a criação da Divisão de Química Ambiental (DAmb) em 1994, houve um workshop em 2001 durante a 24 . Reunião da Sociedade Brasileira de Química, com a finalidade de discutir o Ensino de Química Ambiental no Brasil e ressaltar a necessidade destes materiais.

Nos anos que se seguiram, a disciplina de Química Ambiental passou a ser considerada quase que obrigatória nos cursos de Química e Licenciatura em Química, principalmente após o reconhecimento da importância da questão ambiental na Lei de Diretrizes e Bases da Educação Nacional (LDB 9394/96), onde se ressalta que o estudo do ambiente natural é fundamental para a educação básica, e a publicação dos Parâmetros Curriculares Nacionais do Ensino Médio em 1999 que incluía a temática ambiental como um dos temas transversais no ensino. ${ }^{199}$

A partir daí, surgem os primeiros livros de Química Ambiental traduzidos para o português, a Química Nova na Escola publicou inúmeros artigos relacionados à Química Ambiental que enriquecem o ensino tanto na Educação Básica como Superior, além dos Cadernos Temáticos versando especialmente sobre vários temas de Química Ambiental. Três Cadernos já foram publicados ${ }^{200}$, sendo o primeiro chamado de Química Ambiental (no. 1, 2001), o segundo, Química, Vida e Ambiente (no.5, 2003) e finalmente o terceiro, Recursos Minerais, Água e Meio Ambiente (no. 8, 2014). Materiais digitais também foram disponibilizados na forma de páginas de internet, jogos eletrônicos, objetos de aprendizagem, ressaltando a questão ambiental tanto para ensinar Química Ambiental, ou mesmo ensinar química através da Química Ambiental.

Com o avanço na formação de doutores e mestres na área de Química Ambiental, houve um aumento também dos cursos de graduação em Química Ambiental. Na graduação, o MEC mantém em atividade, 7 cursos de Química Ambiental no Brasil, sendo 2 de caráter tecnológico e os demais de bacharelado, sendo que dois deles são de bacharel em Química com ênfase em Química Ambiental e são oferecidos pela USP. Dos 7 cursos, 2 são oferecidos por instituições privadas. ${ }^{201}$ Embora os cursos de química ambiental sejam fruto dos próprios associados da Sociedade Brasileira de Química, a sua interdisciplinaridade fez com que houvesse um aumento de cursos de graduação em Ciências Ambientais, os quais muitas vezes estão ligados a outras áreas que não a Química. No MEC, estão cadastrados 8 cursos em Ciências Ambientais e 1 em Ciências Biológicas com ênfase em Ciências Ambientais. Na pós-graduação, não há programas denominados diretamente de Química Ambiental. Geralmente, a Química Ambiental encontra-se como disciplina integrada em programas de Química, sendo que o único que tem ao menos como área básica é a UNESP (campus São José do Rio Preto), cadastrado como Análise de traços e Química Ambiental. ${ }^{202} \mathrm{O}$ número de mestrados na área de conhecimento de Ciências Ambientais em funcionamento em 2017 é de 84 e de doutorado é de 42, tendo também 37 cursos de mestrado profissional. Os inúmeros químicos com formação na área de Química Ambiental estão atuando nestes diversos cursos pelo Brasil.

\section{CONSIDERAÇÕES FINAIS}

Falar da atuação dos Químicos no Ambiente não é tarefa simples, pois o alcance é tão grande que ultrapassa muito a fronteira do que foi relatado neste artigo, no qual procurou-se dar ênfase aos processos químicos que ocorrem nos diferentes compartimentos ambientais, por meio de influências antrópicas ou não. Porém, há que se ressaltar que muitos Químicos Ambientais têm tido papel importante na minimização dos efeitos de diferentes espécies químicas presentes no ambiente, não só com a identificação dos problemas ambientais, mas também na produção de tecnologias para o tratamento de diferentes poluentes em águas, solos e atmosferas. A atuação dos Químicos Ambientais no Brasil também tem sido importante nas alterações e mudanças de legislações vigentes ou mesmo nos cuidados com o ambiente após a detecção e avaliação ambiental envolvendo determinados contaminantes. Porém não menos importante, é a atuação de químicos em temas relacionados à Energia, onde os estudos sobre impactos da matriz energética atual, os trabalhos de utilização de energias renováveis, eficiência energética e a grande preocupação com as mudanças climáticas, não se completa sem a atuação de químicos preparados para estes temas.

Cabe ainda ressaltar alguns dos desafios que a Química Ambiental no Brasil vem enfrentando. Observa-se, por exemplo, que um grande número de dados e de estudos já permite ter informações consistentes sobre as fontes, as transformações e o destino de vários poluentes em ambientes de regiões temperadas, pelo menos para alguns já estudados de longa data como metais, nutrientes e alguns pesticidas. Porém, no Brasil, informações quantitativas sobre fontes são ainda frequentemente escassas ou de difícil acesso. Além disso, o número de estudos sobre os impactos dos fatores ambientais específicos de regiões tropicais e subtropicais (temperatura, pluviometria, natureza dos solos, intensidade da radiação solar, etc.) sobre a transformação das substâncias químicas nos diferentes compartimentos é muitas vezes limitado. O desafio vem crescendo quando se consideram os contaminantes emergentes para os quais nem sempre se tem ainda conhecimento completo das suas propriedades físico-químicas e sobre sua reatividade, como no caso das nanopartículas manufaturadas. Como ressaltado anteriormente, a presença destas substâncias em concentrações na escala micro ou nano, constitui um outro amplo desafio para estudos ambientais. Neste contexto, acreditamos que a organização de redes temáticas em escala nacional ou regionais incluindo químicos e pesquisadores de outras áreas com a finalidade de garantir a interdisciplinaridade necessária, deve permitir um avanço significativo da Química Ambiental no Brasil.

Na perspectiva do ensino e do estudo da Química Ambiental é importante ressaltar que esta disciplina não tem como objetivo ensinar apenas o que é poluição antrópica e seus efeitos, mas fazer com que o estudante tenha conhecimento dos processos químicos que ocorrem no ambiente ao seu redor, e que os mesmos não podem ser vistos de forma isolada e sem integração com o passado, presente e futuro.

Por fim, com base em todo o exposto neste artigo, é possível afirmar que a Química Ambiental brasileira vem sendo desenvolvida com suficiente abrangência e profundidade, com qualidade, e buscando cada vez a tão almejada internacionalização, contribuindo decisiva e concretamente para a construção do conhecimento e igualmente para a formação de recursos humanos, em uma área carente e frequentemente negligenciada pelo Estado, de maneira geral.

\section{REFERÊNCIAS}

1. De Andrade, J. B.; Quim. Nova 1992, 15, 173.

2. Mors, W. B.; Avaliação \& Perspectivas, CNPq; 1982.

3. Mozeto, A. A.; Jardim, W. de F.; Quim. Nova 2002, 25, 7.

4. Marques, A. J.; Filgueiras, C. A. L.; Quim. Nova 2010, 33, 1612.

5. CETESB Histórico - http://ar.cetesb.sp.gov.br/ acessado em junho de 2017.

6. Trindade, H. A.; Pfeiffer, W. C.; Londres, H.; Costa-Ribeiro, C. L.; Environ. Sci. Technol. 1981, 15, 84.

7. Orsini, C. Q.; Artaxo Netto, P.; Tabacniks, M. H.; Atmos. Environ. 1982, 16, 2177.

8. Orsini, C. Q.; Tabacniks, M. H.; Artaxo, P.; Andrade, M. F.; Kerr, A.S.; Atmos. Environ. 1986, 20, 2259. 
9. Carvalho, F. M.; Barreto, M. L.; Silvany-Neto, A. M.; Waldron, H. A.; Tavares, T. M.; Sci. Total Environ. 1984, 35, 71.

10. Carvalho, F.; Tavares, T. M.; Souza, S. P.; Souza, S. P.; Linhares, P. S.; Environ. Res. 1984, 33, 300.

11. Pfeiffer, W. C.; Lacerda, L. D.; Environ. Technol. Lett. 1988, 9, 325.

12. Martinelli, L. A.; Ferreira, J. R.; Forsberg, B. R.; Victoria, R. L.; Ambio, 1988, 17, 252.

13. De Andrade, J. B.; Miguel, A. H.; Int. J. Environ. Anal. Chem. 1985, 21, 229.

14. Miguel, A. H.; De Andrade J. B; Hering, S. V.; Int. J. Environ. Anal. Chem, 1986, 26, 265.

15. Tanner, R. L.; Miguel, A. H.; Andrade, J. A.; Gaffney, J. S.; Strett, G. E.; Environ. Sci. Technol. 1988, 22, 1026.

16. Artaxo, P.; Maenhaut, W.; Storms, H.; Vangrieken, R.; J. Geophys. Res.: Atmos. 1990, 95, 16971.

17. Miguel, A. H.; Environ. Sci. Technol. 1991, 25, 590.

18 Harriss, R. C.; Garstang, M.; Wofsy, S. C.; Beck, S. M.; Bendura, R. J.; Coelho, J. R. B.; Drewry J. W.; Hoell Jr., L J.; Matson, P. A.; Mcneil, R. J. L.; Molion, C. B.; Navarro, R. L.; Rabine, V.; Snell, D. R. L.; J. Geophys. Res.; 1990, 95, 16721.

19 De Andrade, J. B.; Miguel, A. H.; Quim. Nova, 1985, 8, 356.

20 Cardoso, A. A.; Pitombo, L. R. M.; Quim. Nova, 1989, 12, 148.

21 Miguel, A. H.; Quim. Nova, 1991, 14(4Sup), 25.

22 Cardoso, A. A.; Quim. Nova, 1991, 14(4Sup), 19.

23 Fornaro, A.; Isolani, P. C.; Gutz, I. G. R.; Atmos. Environ, 1993, 27B, 307.

24 Campos, V. P.; Cruz, L. P. S.; Godoi, R. H. M.; Godoi, A. F. L.; Tavares, T. M.; Microchem. J. 2010, 96, 132.

25 Santos, A. G.; Regis, A. C. D.; da Rocha, G. O.; Bezerra, M. A.; de Jesus, R. M.; De Andrade, J. B.; J. Chromatogr, 2016, 1435, 6.

26 Alonso, C. D.; Martins, M. H. R. B.; Romano, J.; Godinho, R.; J. Air Waste Manage. Assoc. 1997, 47, 1297.

27 Allen, A. D.; McGonigle, A. J. S.; Cardoso, A. A.; Machado, C. M. D.; Davison, B.; Paterlini, W. C.; Rocha, G. O.; De Andrade, J. B.; J. Braz. Chem. Soc., 2009, 20, 214.

28 Campos, V. P.; Couto, E. R.; Miranda, J. P.; Almeida, A. S.; Cruz, L. P. S.; Barbosa, J. L.; Tavares, T. M.; Microchem. J. 2013, 109, 58.

29 Sousa, S. I. V.; Pires, J. C. M.; Martins, E. M.; Fortes, J. D. N.; AlvimFerraz, M. C. M.; Martins, F.G.; Environment International, 2012, 44, 18 .

30 Vieira-Filho, M. S.; Lehmann, C., Fornaro, A.; Atmos. Environ. 2015, 101, 200.

31 Gioda, A.; Ventura, L. M. B.; Ramos, M. B.; Silva, M. P. R.; Water, Air Soil Pollut. 2016, 227, 86.

32 Carvalho, C. N.; Leprun, J.C.; Biogeochemistry, 1991, 14, 99.

33 Forti, M. C.; Carvalho, A; Melfi, A. J.; Montes, C. R. Atmos. Environ. (1994), 2001, 130, 1121.

34 de Mello, W. Z.; Environ. Pollut. 2001, 114, 235.

35 Fornaro, A.; Gutz, I.; Atmos. Environ. 2006, 40, 5893.

36 Gonçalves, C.; dos Santos, M. A.; Fornaro, A.; Pedrotti, J. J.; J. Braz. Chem. Soc., 2010, 21, 331.

37 Sato, M. I. Z.; Valent, G. U.; Coimbrão, C. A.; Coelho, M. C. L. S.; Sanchez, P. S.; Alonso, C. D.; Martins, M. T.; Mutat. Res. 1995, 335, 317.

38 Saldiva, P. H. N.; Pope, C. A.; Schwartz, J.; Dockery, D. W.; Lichtenfels, A. J.; Salge, J. M.; Barone, I.; Bohm, G. M.; Arch. Environ. Health, 1995, 50, 159 .

39 de Freitas, C. U.; de Leon, A. P.; Junger, W.; Gouveia, N.; Rev. Saúde Pública, 2016, 50, 4

40 Ferreira, T.; Forti, M.; de Freitas, C. U.; Nascimento, F.; Junger, W.; Gouveia, N.; Int. J. Environ. Res. Public Health, 2016, 13, 947.

41 Yoshizaki, K.; Fuziwara, C. S.; Brito, J. M.; Santos, T. M. N.; Kimura, E. T.; Correia, A. T.; Amato-Lourenco, L. F.; Vasconcellos, P.; Silva, L. F.;
Brentani, M. M.; Mauad, T.; Saldiva, P. H. N.; Macchione, M. Environ. Pollut. 2016, 213, 359.

42 Pimentel, A. S.; Arbilla, G.; Quim. Nova, 1997, 20, 252.

43 Guimarães, C. S.; Varandas, L. S.; Arbilla, G.; J. Braz. Chem. Soc., 2010, 21,481 .

44 Sousa, E. T.; Lopes, W. A.; de Andrade, J. B.; Quim. Nova, 2016, 39 , 486.

45 Da Silva, C. M.; Souza, E. C. C. A.; Da Silva, L. L.; Oliveira, R. L.; Corrêa, S. M.; Arbilla, G.; Bull. Environ. Contam. Toxicol. 2016, 97, 653.

46 Pinto, J. P.; Solci, M. C.; J. Braz. Chem. Soc., 2007, 21, 481.

47 Nogueira, T.; Dominutti, P. A.; De Carvalho, L. R. F.; Fornaro, A.; Andrade, M. F.; Fuel (Guildford), 2014, 134, 505.

48 Casal, C. S.; Arbilla, G.; Corrêa, S. M.; Atmos. Environ. (1994), 2014, 96, 107.

49 Corrêa, S. M.; Arbilla, G.; Marques, M. R. Da C.; Atmos. Pollut. Res. 2012, 3, 163. 2012.

50 Silva, C. M.; Silva, L. L.; Corrêa, S. M.; Arbilla, G.; Bull. Environ. Contam. Toxicol. 2016, 97, 781.

51 Lopes Oliveira, R.; Varandas, L.; Arbilla, G.; Atmos. Pollut. Res. 2014, $5,87$.

52 Vasconcellos, P. C.; Zacarias, D.; Magalhães, D.; Rocha, G.; Wat. Air Soil Poll. 2011, 222, 325.

53 Guarieiro, A. L. N.; Santos, J. V. S.; Eiguren-Fernandez, A.; Torres. E. A.; Rocha, G. O.; Andrade, J. B.; Fuel, 2014, 116, 490.

54 Urban, R. C.; Alves, C. A.; Allen, A. G.; Cardoso, A. A.; Campos, M. L. A. M.; Atmos. Res. 2016, 169, 271.

55 Souza, K. F.; Carvalho, L. R. F.; Allen, A. G.; Cardoso, A. A.; Atmos. Environ. (1994), 2014, 83, 193.

56 Programa LBA (Experimento de Larga Escala da Biosfera-Atmosfera na Amazônia), http://lba2.inpa.gov.br/, acessado em junho de 2017.

57 Formenti, P.; Andreae, M. O.; Lange, L.; Roberts, G.; Cafmeyer, J.; Rajta, I.; Maenhaut, W.; Holben, B. N.; Artaxo, P.; Lelieveld, J.; J. Geophys. Res. 2011, 06, 14919.

58 Andreae, M. O.; Artaxo, P.; Brandão, C.; Carswell, F. E.; Ciccioli, P.; da Costa, A. L.; Culf, A. D.; Esteves, J. L.; Gash, J. H. C.; Grace, J.; Kabat, P.; Lelieveld, J.; Malhi, Y.; Manzi, A. O.; Meixner, F. X.; Nobre, A. D.; Nobre, C.; Ruivo, M. D. L. P.; Silva-Dias, M. A.; Stefani, P.; Valentini, R.; Jouanne, J. V.; Waterloo, M. J. J. Geophys. Res, 2002, 107, 8066.

59 Kirchhoff, V. W. J. H.; Setzer, A. W.; Pereira, M. C.; Geophys. Res. Lett. 1989, 16, 469 .

60 Kirchhoff, V. W. J. H.; Marinho, E. V. A.; Atmos. Environ. 1994, $28,69$.

61 Kesselmeier, J.; Kuhn, U.; Wolf, A.; Ciccioli, P.; Braancaleoni, E.; Frattoni, M.; Guenther, A.; Greenberg, J.; Vasconcellos, P. C.; Oliva, T.; Tavares, T.; Artaxo, P.; Atmos. Environ. 2000, 34, 4063.

62 de Oliveira Alves, N.; Brito, J.; Caumo, S.; Arana, A.; De Souza H. S.; Artaxo, P.; Hillamo, R.; Teinilä, K.; Batistuzzo, S. R. M.; Vasconcellos, P. C.; Atmos. Environ. (1994), 2015, 120, 277.

63 Gonçalves, C.; Figueiredo, B. R.; Alves, C. A.; Cardoso, A. A; Da Silva, R.; Kanzawa, S. H.; Vicente, A. M.; Atmos. Res. 2016, 182, 102.

64 Brickus, L. S. R.; Aquino Neto, F. R.; Quim. Nova, 1999, 22, 65.

65 Grosjean, D.; Miguel, A. H.; Tavares, T. M.; Atmos. Environ. 1990, 24B, 101.

66 Allen, A. G.; Miguel, A. H.; Atmos. Environ. 1995, 29, 3519

67 Miguel, A. H.; Aquino Neto, F. R.; Cardoso, J. N.; Vasconcellos, P. C.; Pereira, A. S.; Marquez, K. S. G.; Environ. Sci. Technol. 1995, 29, 338.

68 Santos, C. Y.; Aquino Neto, F. R.; Cardoso, J. N.; Indoor Built Environ. 1997, 6, 168.

69 Gioda, A.; Aquino Neto, F. R.; Quim. Nova, 2003, 26, 359.

70 Cavalcante, R. M.; Seyffert, B. H.; D’Ocac, M. G. M.; Nascimento, R. F.; Campelo, C. S.; Pinto, I. S.; Anjos, F. B.; Costa, A. H. R.; Indoor Built Environ. 2005, 14, 165. 
71 Sousa, F. W.; Caracas, I. B.; Nascimento, R.; Cavalcante, R. M., Build Environ. 2011, 46, 2115.

72 Martins, E. M.; de Sa Borba, P. F.; dos Santos, N. E.; Reis, P. T. B.; Silveira, R. S.; Correa, S. M.; Environ. Monit. Assess., 2016, 188, 608.

73 Amador, I. R.; Pinto, J. P.; Solci, M. C.; Orbital: Electron. J. Chem. 2016, 8, 189.

74 Segalin, B.; Kumar, P.; Micadei, K.; Fornaro, A.; Gonçalves, F. L. T.; Atmos. Environ. 2017, 148, 139.

75 Godoi, R. H. M.; Avilo Jr. D.; Campos, V. P.; Tavares, T. M.; de Marchi, M. R. R.; Van Grieken, R.; Godoi, A. F. L.; Wat. Air Soil Pollut., 2009, 9, 171.

76 Godoi, R. H. M.; Godoi, A. F. L.; Gonçalves Jr., S. J.; Paralovo, S. L.; Borillo, G. C.; Barbosa, C. G. G.; Arantes, M. G.; Charello, R. C.; Rosário Filho, N. A.; Grassi, M. T.; Yamamoto, C. I.; Potgieter-Vermaak, S.; Rotondo, G. G.; De Wael. K.; van Grieken, R.; Sci. Total Environ. 2013, 463-464, 639.

77 Gioda, A.; Aquino Neto, F. R.; Indoor Built Environ. 2007, 16, 371.

78 Siqueira, C.; Gioda, A.; Carneiro, F. P.; Ramosa, M. C. K. V.; Aquino Neto, F. R.; J. Braz. Chem. Soc. 2011, 22, 2127.

79 Castro, B. P.; Machado, G. S.; Bauerfeldt, G. F.; Atmos. Environ. 2015, 104, 22.

80 Sabino, F.C.; Pinto, J. P.; Amador, I. R.; Martins, L. D.; Solci, M. C.; J. Braz. Chem. Soc. 2016, 9, 1551.

81 Lara, W. H.; Barreto, H. H. C.; Rev.Inst.Adolfo Lutz 1972, 32, 1.

82 Ferreira, J. R.; Ambio 1979, 8, 210.

83 Pfeiffer, W. C.; Lacerda, L. D.; Malm, O.; Souza, C. M. M.; da Silveira, E. G.; Bastos, W. R.; Sci. Total Environ. 1989, 87-88, 233.

84 Lacerda, L. D.; Pfeiffer, W. C.; Marins, R. V.; Rodrigues, S.; Souza, C. M. M.; Bastos, W. R.; Water. Air. Soil Pollut. 1991, 55, 513.

85 Filho, R. S.; Maddock, J. E. L.; J. Geochemical Explor. 1997, 58, 231.

86 Porvari, P.; Sci. Total Environ. 1995, 175, 109.

87 Lacerda, L. D.; Water. Air. Soil Pollut. 1997, 97, 247.

88 Guimarães, J. R. D.; Fostier, A.-H.; Forti, M. C.; Melfi, J. A.; Kehrig, H.; Mauro, J. B. N.; Malm, O.; Krug, J. F.; Ambio 1999, 28, 296.

89 Fostier, A.-H.; Forti, M. C.; Guimarães, J. R. D.; Melfi, A. J.; Boulet, R.; Espirito Santo, C. M.; Krug, F. J.; Sci. Total Environ. 2000, 260, 201.

90 Fadini, P. S.; Jardim, W. F.; Sci. Total Environ. 2001, 275, 71.

91 Lechler, P. J.; Miller, J. R.; Lacerda, L. D.; Vinson, D.; Bonzongo, J.-C.; Lyons, W. B.; Warwick, J. J.; Sci. Total Environ. 2000, 260, 87.

92 Kehrig, H. D. A.; Costa, M.; Moreira, I.; Malm, O.; Environ. Sci. Pollut. Res. 2001, 87, 275.

93 Guimarães, J. R. D.; Meili, M.; Hylander, L. D.; Silva, E. D. C. E.; Roulet, M.; Mauro, J. B. N.; De Lemos, R. A.; Sci. Total Environ. 2000, 261, 99.

94 Pfeiffer, W. C.; Fiszman, M.; Malm, O.; Maurício Azcue, J.; Sci. Total Environ. 1986, 58, 73.

95 Fernandes, H. M.; Bidone, E. D.; Veiga, L. H. S.; Patchineelam, S. R.; Environ. Pollut. 1994, 85, 259.

96 de Lacerda, L. D.; Pfeiffer, W. C.; Fiszman, M.; Sci. Total Environ. 1987, 65, 163.

97 Jordão, C. P.; Pereira, J. L.; Jham, G. N.; Bellato, C. R.; Environ. Technol. 1999, 20, 489.

98 Jordão, C. P.; Pereira, J. L.; Jham, G. N.; Sci. Total Environ. 1997, 207, 1.

99 Sodré, F. F.; Dos Anjos, V. E.; Prestes, E. C.; Grassi, M. T.; J. Environ. Monit. 2005, 7, 581.

100 Sodré, F. F.; Grassi, M. T.; Water. Air. Soil Pollut. 2007, 178, 103.

101 Prestes, E. C.; Dos Anjos, V. E.; Sodré, F. F.; Grassi, M. T.; J. Braz. Chem. Soc. 2006, 17, 53.

102 De Freitas Melo, V.; De Andrade, M.; Batista, A. H.; Favaretto, N.; Grassi, M. T.; De Campos, M. S.; Quim. Nova 2012, 35, 22.

103 Matschullat, J.; Perobelli Borba, R.; Deschamps, E.; Figueiredo, B. R.; Gabrio, T.; Schwenk, M.; Appl. Geochemistry 2000, 15, 181.
104 Magalhães, V. F.; Carvalho, C. E. V.; Pfeiffer, W. C.; Water. Air. Soil Pollut. 2001, 129, 83.

105 Borba, R. P.; Figueiredo, B. R.; Matschullat, J.; Environ. Geol. 2003, 44, 39.

106 Figueiredo, B. R.; Borba, R. P.; Angélica, R. S.; Environ. Geochem. Health 2007, 29, 109.

107 Mirlean, N.; Andrus, V. E.; Baisch, P.; Griep, G.; Casartelli, M. R.; Mar. Pollut. Bull. 2003, 46, 1480.

108 Hatje, V.; Macedo, S. M.; de Jesus, R. M.; Cotrim, G.; Garcia, K. S.; de Queiroz, A. F.; Ferreira, S. L. C.; Mar. Pollut. Bull. 2010, 60, 2225.

109 dos Anjos, V. E.; da C. Machado, E.; Grassi, M. T.; Aquat. Geochemistry 2012, 18, 407.

110 Silva, L. F. O.; Fdez-Ortiz de Vallejuelo, S.; Martinez-Arkarazo, I.; Castro, K.; Oliveira, M. L. S.; Sampaio, C. H.; de Brum, I. A. S.; de Leão, F. B.; Taffarel, S. R.; Madariaga, J. M.; Sci. Total Environ. 2013, 447, 169.

111 Rezende, P. S.; Costa, L. M.; Windmöller, C. C.; Arch. Environ. Contam. Toxicol. 2015, 68, 588.

112 Gontijo, E. S. J.; Watanabe, C. H.; Monteiro, A. S. C.; Tonello, P. S.; da Silva, G. A.; Friese, K.; Roeser, H. M. P.; Rosa, A. H.; Chemosphere 2016, 164, 290.

113 Pereira Netto, A. D.; Sisinno, C. L. S.; Moreira, J. C.; Arbilla, G.; Dufrayer, M. C.; Bull. Environ. Contam. Toxicol. 2002, 68, 1.

114 Gomes, A. D. O.; Azevedo, D. D. A.; J. Braz. Chem. Soc. 2003, 14, 358.

115 Meniconi, M. F. G.; Massone, C. G.; Scofield, A. L. In Environment Canada Arctic and Marine Oil Spill Program Technical Seminar (AMOP) Proceedings; 2003; 26, 1.

116 Barbosa, M. C.; De Almeida, M. D. S. S.; Mariz, D. F.; De Almeida, J. L. D. S. S.; J. Hazard. Mater. 2004, 110, 29.

117 Medeiros, P. M.; Bícego, M. C.; Mar. Pollut. Bull. 2004, 49, 761.

118 Medeiros, P. M.; Bícego, M. C.; Castelao, R. M.; Del Rosso, C.; Fillmann, G.; Zamboni, A. J.; Environ. Int. 2005, 31, 892.

119 Da Silva, T. F.; Azevedo, D. D. A.; Neto, F. R. D. A.; J. Braz. Chem. Soc. 2007, 18, 628.

120 Farias, C. O.; Hamacher, C.; Wagener, A. d. L. R.; Scofield, A. d. L.; Org. Geochem. 2008, 39, 289.

121 Meire, R. O.; Azeredo, A.; Pereira, M. d. S.; Torres, J. P. M.; Malm, O.; Chemosphere 2008, 73, 1.

122 Pietzsch, R.; Patchineelam, S. R.; Torres, J. P.; M. Mar. Chem. 2010, $118,56$.

123 da Silva, D. A. M.; Bícego, M. C.; Mar. Environ. Res. 2010, 69, 277.

124 Maioli, O. L. G.; Rodrigues, K. C.; Knoppers, B. A.; Azevedo, D. A.; J. Braz. Chem. Soc. 2010, 21, 1116.

125 Leite, N. F.; Peralta-Zamora, P.; Grassi, M. T.; J. Environ. Sci. 2011, 23 (6), 904.

126 Martins, C. C.; Bícego, M. C.; Mahiques, M. M.; Figueira, R. C. L.; Tessler, M. G.; Montone, R. C.; Mar. Pollut. Bull. 2011, 63, 452.

127 Wagener, A. D. L. R.; Meniconi, M. D. F. G.; Hamacher, C.; Farias, C. O.; da Silva, G. C.; Gabardo, I. T.; Scofield, A. D. L.; Mar. Pollut. Bull. 2012, 64, 284.

128 Lima, M. B.; Feitosa, E. A.; Emídio, E. S.; Dórea, H. S.; Alexandre, M. R.; Mar. Pollut. Bull. 2012, 64, 1721.

129 de Abreu-Mota, M. A.; de Moura Barboza, C. A.; Bícego, M. C.; Martins, C. C.; Chemosphere 2014, 103, 156.

130 Frena, M.; Bataglion, G. A.; Sandini, S. S.; Kuroshima, K. N.; Eberlin, M. N.; Madureira, L. A. S.; J. Braz. Chem. Soc. 2017, 28, 603.

131 Venturini, N.; Tommasi, L. R.; Mar. Pollut. Bull. 2004, 48, 97.

132 Froehner, S.; MacEno, M.; Da Luz, E. C.; Souza, D. B.; MacHado, K. S.; Environ. Monit. Assess. 2010, 168, 205.

133 Sparovek, G.; Anisimova, M. A.; Kolb, M.; Bahadir, M.; Wehage, H.; Schnug, E.; J. Environ. Qual. 2001, 30, 2006.

134 Souza Brito, E. M.; Reis Vieira, E. D.; Machado Torres, J. P.; Malm, O.; Quim. Nova 2005, 28, 941. 
135 Rissato, S. R.; Galhiane, M. S.; Ximenes, V. F.; de Andrade, R. M. B.; Talamoni, J. L. B.; Libânio, M.; de Almeida, M. V.; Apon, B. M.; Cavalari, A. A.; Chemosphere 2006, 65, 1949.

136 Barra, R.; Colombo, J. C.; Eguren, G.; Gamboa, N.; Jardim, W. F.; Mendoza, G.; Persistent organic pollutants (POPs) in eastern and western South American countries; 2006, 185.

137 Yogui, G. T.; Santos, M. C. O.; Bertozzi, C. P.; Montone, R. C.; Mar Pollut. Bull. 2010, 60, 1862.

138 Fonseca, E. M.; Baptista Neto, J. A.; Pereira, M. P. S.; Silva, C. G.; Arantes Junior, J. D.; Mar. Pollut. Bull. 2014, 82, 45.

139 Oliveira, A. H. B.; Cavalcante, R. M.; Duaví, W. C.; Fernandes, G. M.; Nascimento, R. F.; Queiroz, M. E. L. R.; Mendonça, K. V.; Sci. Total Environ. 2016, 542, 254

140 Mendez, A.; Ng, C. A.; Torres, J. P. M.; Bastos, W.; Bogdal, C.; dos Reis, G. A.; Hungerbuehler, K.; Environ. Sci. Pollut. Res. 2016, 23 , 10317.

141 Almeida, F. V.; Centeno, A. J.; Bisinoti, M. C.; Jardim, W. F.; Quim. Nova 2007, 30, 1976.

142 Fernandez, M. A.; Limaverde, A. M.; de Castro, I. B.; Almeida, A. C.; de Luca Rebello Wagener, A.; Cad. Saude Publica 2002, 18, 463.

143 Godoi, A. F. L.; Montone, R. C.; Santiago-Silva, M.; J. Chromatogr. A 2003, 985, 205.

144 Fernandez, M. A.; Wagener, A. D. L. R.; Limaverde, A. M.; Scofield, A L.; Pinheiro, F. M.; Rodrigues, E.; Mar. Environ. Res. 2005, 59, 435.

145 Santos, D. M.; Araújo, I. P.; Machado, E. C.; Carvalho-Filho, M. A. S.; Fernandez, M. A.; Marchi, M. R. R.; Godoi, A. F. L.; Mar. Pollut. Bull. 2009, 58, 1926.

146 Pletsch, A. L.; Beretta, M.; Tavares, T. M.; Quim. Nova 2010, 33, 451.

147 Santos, D. M. d.; Sant'Anna, B. S.; Sandron, D. C.; Cardoso de Souza, S.; Cristale, J.; Marchi, M. R. R. d.; Turra, A.; Estuar. Coast. Shelf Sci. 2010, 88,322 .

148 de Oliveira, C. R.; dos Santos, D.; dos Santos Madureira, L. A.; de Marchi, M. R. R.; J. Hazard. Mater. 2010, 181, 851.

149 De Castro, I. B.; Perina, F. C.; Fillmann, G.; Environ. Monit. Assess. 2012, 184,1781.

150 Castro, I. B.; Rossato, M.; Fillmann, G.; Environ. Toxicol. Chem. 2012 $31,947$.

151 dos Santos, D. M.; Turra, A.; de Marchi, M. R. R.; Montone, R. C.; Environ. Sci. Pollut. Res. 2016, 23, 16152.

152 Montagner, C. C.; Jardim, W. F.; J. Braz. Chem. Soc. 2011, 22, 1452.

153 Thomas, K. V.; Araújo da Silva, F. M.; Langford, K. H.; de Souza, A. D. L.; Nizzeto, L.; Waichman, A. V.; J. Am. Water Resour. Assoc. 2014, 50, 302.

154 Beretta, M.; Britto, V.; Tavares, T. M.; da Silva, S. M. T.; Pletsch, A. L.; J. Soils Sediments 2014, 14, 1278.

155 de Sousa, D. N. R.; Mozeto, A. A.; Carneiro, R. L.; Fadini, P. S.; Sci. Total Environ. 2014, 484, 19.

156 Campanha, M. B.; Awan, A. T.; de Sousa, D. N. R.; Grosseli, G. M.; Mozeto, A. A.; Fadini, P. S.; Environ. Sci. Pollut. Res. 2014, 22, 7936.

157 Machado, K. C.; Grassi, M. T.; Vidal, C.; Pescara, I. C.; Jardim, W. F.; Fernandes, A. N.; Sodré, F. F.; Almeida, F. V.; Santana, J. S.; Canela, M. C.; Nunes, C. R. O.; Bichinho, K. M.; Severo, F. J. R.; Sci. Total Environ. 2016, 572, 138.

158 Campestrini, I.; Jardim, W. F.; Sci. Total Environ. 2017, 576, 374.

159 Sparks, D.L.; Environmental Soil Chemistry, $2^{\text {nd }}$ ed., Academic Press: San Diego, 2003.

160 Egboka, B. C. E.; Nwankwor, G. I.; Orajaka, I. P.; Ejiofor, A. O., Environ. Health Perspect. 1989, 83, 39.

161 Hamelin, R.; Quim. Nova, 1995, 18, 68.

162 Rebouças, A. C.; Water Sci. Technol. 1991, 24, 141.

163 Amaral, E. C. S.; Vianna, M. E. C.; Godoy, J. M.; Rochedo, E. R. R.; Campos, M. J.; Dorio, M. A. P.; Oliveira, J. P.; Pereira, J. C. A.; Reis, W. G.; Health Physics 1991, 60, 91.
164 Rochedo, E. R. R.; Amaral, E. C. S.; Bartell, S. M.; J. Environ. Radioact. 1992, 15, 171

165 Oliveira, R. M.; Brilhante, O. M.; Moreira, J. C.; Miranda, A. C.; Rev. Saude Publica 1995, 29, 228.

166 Hirata, R. C. A.; Cleary, R. W.; Water Sci.Technol. 1991, 24, 127.

167 Simon, Z.; Tedesco, M. J.; Gianello, C.; Water Sci.Technol. 1991, 24, 19.

168 Juca, J. F. T.; Mariano, M. O. H.; Campello, E. M. B.; Environ. Geotech., Vol 1 1996, 91.

169 Jordao, C. P.; Pereira, J. C.; Brune, W.; Pereira, J. L.; Braathen, P. C.; Environ. Technol. 1996, 17, 489.

170 Amaral, E. C. S.; Azevedo, H. L. P.; Mendonca, A. H.; Sci. Total Environ. 1985, 42, 257.

171 Pfeiffer, W. C.; Malm, O.; Souza, C. M. M.; Delacerda, L. D.; Silveira, E. G.; Bastos, W. R.; For. Ecol. Manage. 1991, 38, 239.

172 Aula, I.; Braunschweiler, H.; Malin, I.; Sci. Total Environ. 1995, 175, 97.

173 Malm, O.; Castro, M. B.; Bastos, W. R.; Branches, F. J. P.; Guimaraes, J. R. D.; Zuffo, C. E.; Pfeiffer, W. C.; Sci. Total Environ. 1995, 175, 127.

174 Hylander, L. D.; Silva, E. C.; Oliveira, L. J.; Silva, S. A.; Kuntze, E. K.; Silva, D. X.; Ambio 1994, 23, 478.

175 Queiroz, M. E. C.; Lancas, F. M.; J. Braz. Chem. Soc. 1997, 8, 1.

176 Wilcke, W.; Lilienfein, J.; Lima, S. D.; Zech, W.; J.Plant Nutr.Soil Sci. 1999, 162, 539.

177 Mirlean, N.; Casartelli, M. R.; Garcia, M. R. D.; Quim. Nova 2002, 25, 191.

178 Matos, A. T.; Costa, L. M.; Fontes, M. P. F.; Martinez, M. A.; Trans. ASAE 1999, 42, 903.

179 Windmoller, C. C.; Wilken, R. D.; Jardim, W. D.; Water Air Soil Pollut. 1996, 89, 399

180 Roulet, M.; Lucotte, M.; Saint-Aubin, A.; Tran, S.; Rheault, I.; Farella, N.; Da Silva, E. D.; Dezencourt, J.; Passos, C. J. S.; Soares, G. S.; Guimarães, J. R. D.; Mergler, D.; Amorim, M.; Sci. Total Environ. 1998, 223, 1.

181 Fostier, A.; Forti, M.; Guimarães, J.; Melfi, A.; Boulet, R.; Santo, C.; Krug, F.; Sci. Total Environ. 2000, 260, 201.

182 Dick, D. P.; Burba, P.; Herzog, H.; J. Braz. Chem. Soc. 1999, 10, 140.

183 Rocha, J. C.; Rosa, A. H.; Furlan, M.; J. Braz. Chem. Soc. 1998, 9, 51.

184 Lancas, F. M.; J. Braz. Chem. Soc. 2003, 14, 183.

185 Rawa-Adkonis, M.; Wolska, L.; Namiesnik, J.; Crit. Ver. Anal. Chem. 2003, 33, 199 .

186 Serudo, R. L.; de Oliveira, L. C.; Rocha, R. C.; Paterlini, W. C.; Rosa, A. H.; da Silva, H. C.; Botero, W. G.; Geoderma 2007, 138, 229.

187 de Mello, J. W. V.; Talbott, J. L.; Scott, J.; Roy, W. R.; Stucki, J.; W. Environ. Sci. Pollut. Res. 2007, 14, 388.

188 Lourencettia, C.; de Marchi, M. R. R.; Ribeiroa, M. L.; Talanta 2008, 77,1 .

189 Bilo, F.; Borgese, L.; Cazzago, D.; Zacco, A.; Bontempi, E.; Guarneri, R.; Bernardello, M.; Attuati, S.; Lazo, P.; Depero, L. E.; Environ. Sci. Pollut. Res. 2014, 21,13208.

190 Sanchís, J.; Oliveira, L. F. S.; de Leão, F. B.; Farré, M.; Barceló, D.; Sci. Total Environ. 2015, 505, 172.

191 Ferreira, F. O.; Silva, C. R.; Rath, S.; J. Chromato A 2016, 1471, 118.

192 Adler, R. R.; Lopez, A. G.; Gloeden, E.; Marker, A.; Contaminated Soil '98 1998, 1-2, 163.

193 CETESB; Relatório de estabelecimento de valores orientadores para solos e águas subterrâneas no estado de São Paulo, CETESB: São Paulo, 2001.

194 CONAMA; Resolução $n^{\circ} 420$, de 28 de dezembro de 2009, DOU nº 249 , Brazil, 2009.

195 Bernhardt, E. S.; Rosi, E. J.; Gessner, M. O.; Front Ecol Environ 2017, doi:10.1002/fee. 1450

196 Vodyanitskii, Y. N.; Yakovlev, A. S. Eurasian Soil Sci., 2016, 49, 560.

197 Rieuwerts, J.S.; Chem. Speciation Bioavailability 2007, 19, 75.

198 Jardim, W.F.; Quim. Nova, 1989, 12, 291. 
199 Brasil. Ministério da Educação. Secretaria de Educação Média e Tecnológica; Parâmetros curriculares nacionais: ensino médio, MEC/ SEMTEC: Brasília, 1999.

200 Química Nova na Escola, Caderno Temático de Química Ambiental, 2001,1; Caderno Temático- Química Vida e Ambiente, 2003, 5, Caderno Temático- Recursos Minerais, Água e Meio Ambiente, 2014, 8.
201 Brasil. Ministério da Educação. http://emec.mec.gov.br/ acesso em fevereiro de 2017.

202 CAPES, Plataforma Sucupira, https://sucupira.capes.gov.br/sucupira/, acesso em junho de 2017. 